\title{
High-throughput screening reveals new glutaminase inhibitor molecules
}

Renna K. E. Costa ${ }^{1,2} \dagger$, Camila Rodrigues $\dagger^{3}$, Jean C. H. Campos ${ }^{1} \dagger$, Luciana Paradela ${ }^{1,2} \dagger$, Marilia M. Dias ${ }^{1}$, Bianca Novaes da Silva ${ }^{1}$, Cyro von Zuben de Valega Negrao ${ }^{1,2}$, Kaliandra de Almeida Gonçalves ${ }^{1}$, Carolline F. R. Ascenção ${ }^{1,2}$, Douglas Adamoski ${ }^{1,2}$, Gustavo Fernando Mercaldi ${ }^{1}$, Alliny C. S. Bastos ${ }^{1}$, Fernanda A. H. Batista ${ }^{1}$, Ana Carolina Figueira ${ }^{1}$, Artur T. Cordeiro ${ }^{1}$, Andre L. B. Ambrosio ${ }^{1}$, Rafael V. C. Guido ${ }^{3 *} \dagger$, Sandra M. G. Dias ${ }^{1 *} \dagger$

Supplementary Information

Supplementary Figure S1

Supplementary Figure S2

Supplementary Figure S3

Supplementary Figure S4

Supplementary Figure S5

Supplementary Figure S6

Supplementary Figure S7

Supplementary Figure S8

Supplementary Figure S9

Supplementary Table S1

Supplementary Table S2

Supplementary Table S3

Supplementary Table S4 


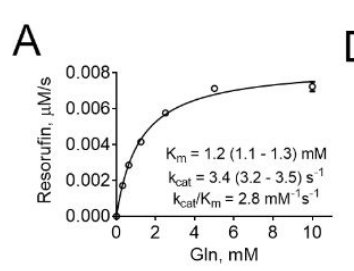

B
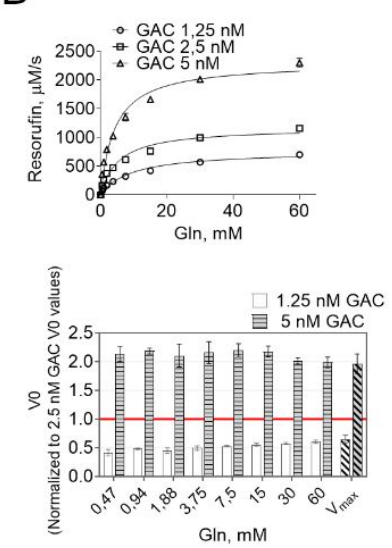
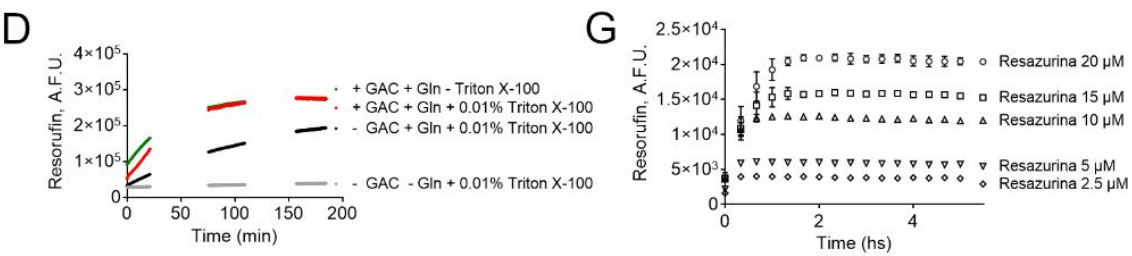

$\mathrm{H}$
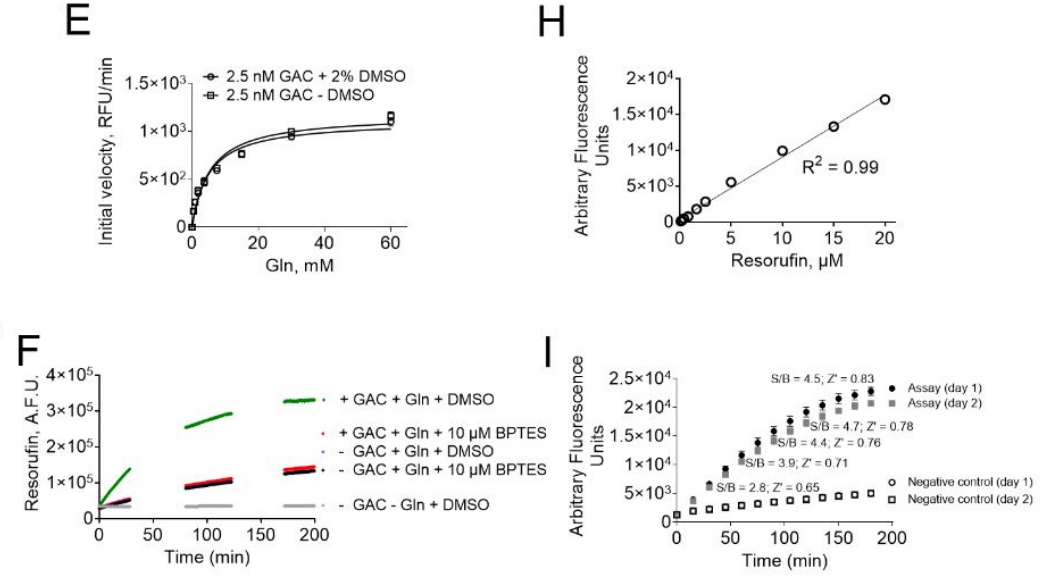

C

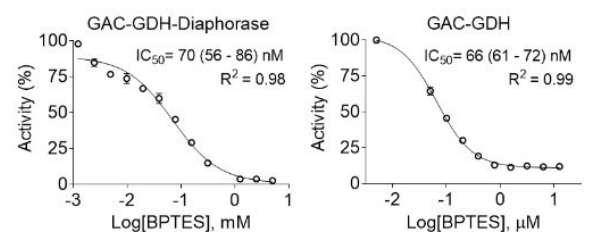

Supplementary Figure S1. Assay optimization. A, Michaelis-Menten of the GAC-GDHDiaphorase fluorescent coupled assay. Obtained $\mathrm{K}_{\mathrm{m}}, \mathrm{k}_{\mathrm{cat}}$ and $\mathrm{k}_{\mathrm{cat}} / \mathrm{K}_{\mathrm{m}}$ for $2.5 \mathrm{nM}$ of enzyme are very close to the same parameters measured for GAC in a GAC-GDH absorbance assay previously published (41) (see table for comparison). $B$, Above, Michaelis-Menten of the GACGDH-Diaphorase fluorescent coupled assay using 1,25 nM and $5 \mathrm{nM}$; below, the ratio between V0 measured for each glutamine concentration (and Vmax) when using 1,25 nM GAC over 2,5 nM GAC (white bars) or $5 \mathrm{nM}$ GAC over 2,5 nM GAC (gray bars), showing the values are proportional to the GAC concentration. The proportionality proves the reaction is driven by GAC. $C$, comparing the $\mathrm{IC}_{50}$ of BPTES over the GAC-GDH-Diaphorase fluorescent coupled assay and the GAC-GDH absorbance assay. D, Measured RFU of the GAC-GDH-Diaphorase fluorescent coupled assay over time showing that $0.01 \%$ Triton $\mathrm{X}-100$ does not affect measurement. $E$, Michaelis-Menten of the GAC-GDH-Diaphorase fluorescent coupled assay showing that $2 \%$ DMSO does not affect the curve qualitatively. $F$, Measured RFU of the GACGDH-Diaphorase fluorescent coupled assay in different conditions over 200 minutes showing 
that either GAC removal $(-\mathrm{GAC}+\mathrm{Gln}+2 \% \mathrm{DMSO})$ or BPTES $10 \mu \mathrm{M}(+\mathrm{GAC}+\mathrm{Gln}+10 \mu \mathrm{M}$ BPTES) treatment gives equivalent measurements. $G$, Measured RFU of the GAC-GDHDiaphorase fluorescent coupled assay over time showing that $20 \mu \mathrm{M}$ resorufin gives the highest signal and best $\mathrm{S} / \mathrm{N}$ (values not shown). H, RFU obtained for different amounts (0 to $20 \mu \mathrm{M}$ ) of the fluorescent product, resorufin, showing the dynamic range of the reaction. $I$, Reproducibility of the GAC-GDH-Diaphorase fluorescent coupled assay over two consecutive days. Z' and S/B for each data point of Assay (day 1).
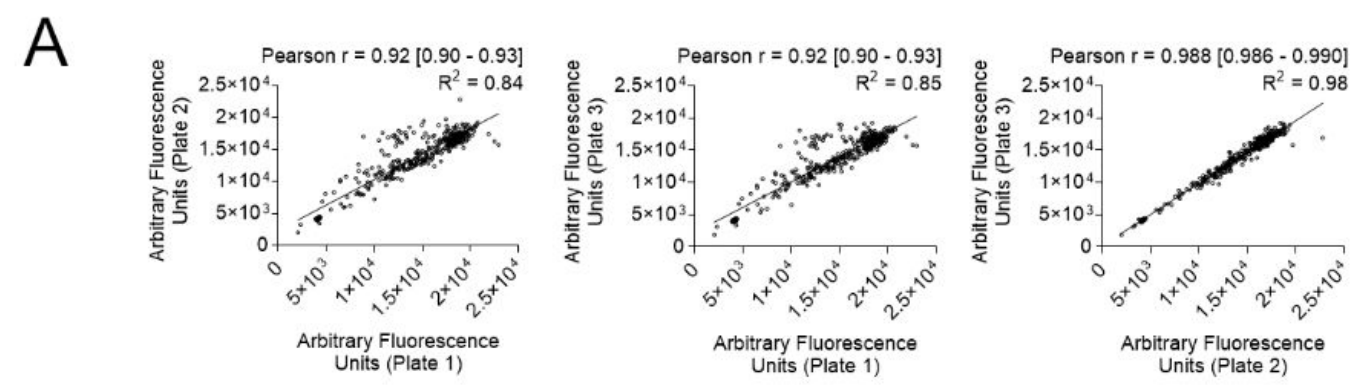

B
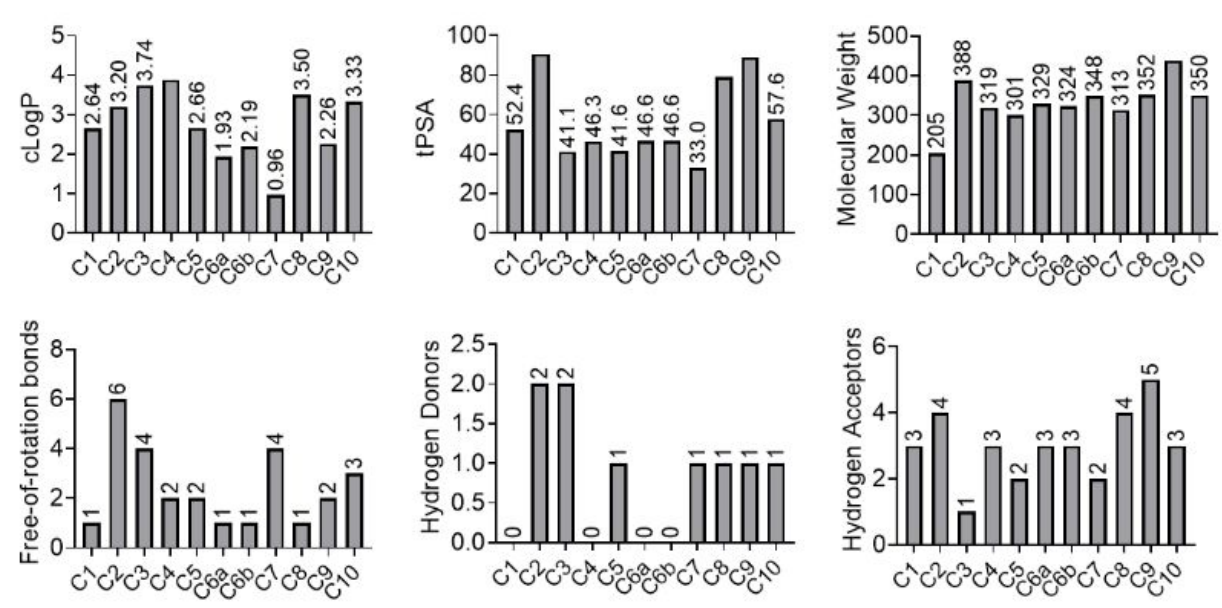

Supplementary Figure S2. A, Confirmation assay of 320 hits was performed in triplicate after cherry-picking. Correlation between plate 1 and 2 (on the left), plates 1 and 3 (in between) and plates 2 and 3 (on the right). Pearson correlation $\mathrm{r}$ and $\mathrm{R}^{2}$ values are shown above each graph. $B$, cLogP, topological polar superficial area (tPSA), Molecular Weight, number of free-ofrotation bonds, number of hydrogen bonds donors and acceptors graphs of the 11 re-supplied compounds. 
A
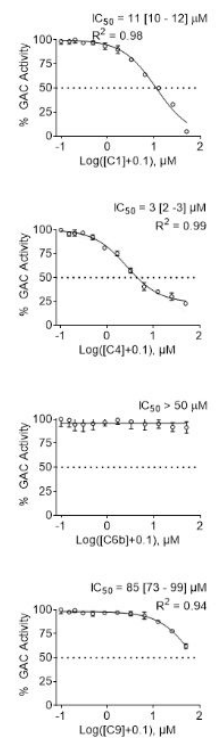
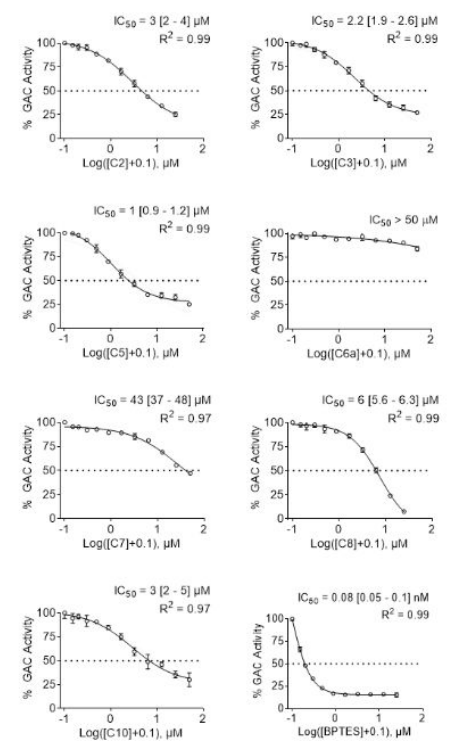

B
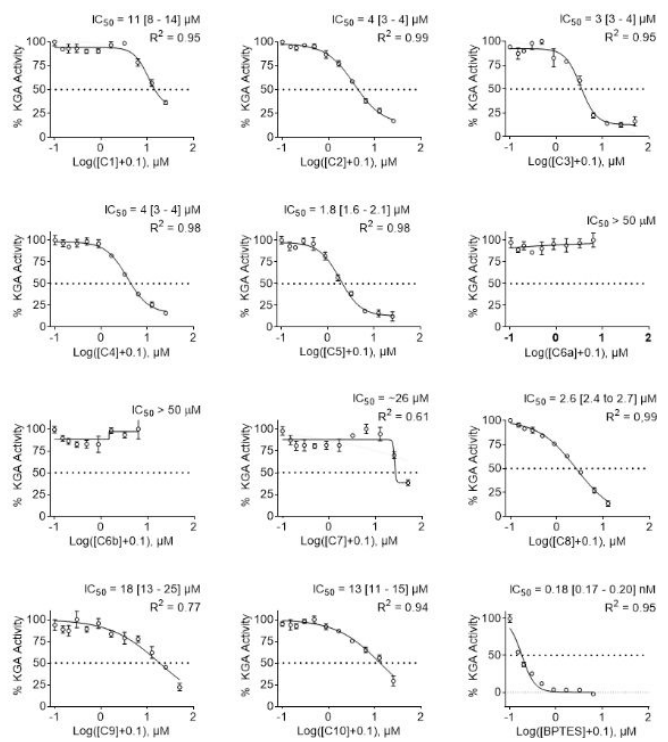

Supplementary Figure S3. A, curves of C1-C10 on the GAC (A) or KGA (B) enzymatic activity. Values within the brackets represents an interval confidence of 95\% (CI 95\%). 
A

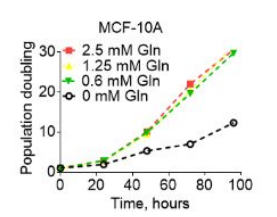

C

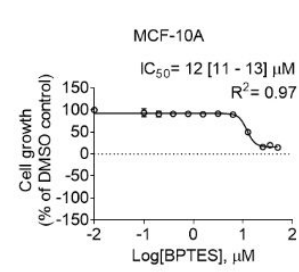

SKBR3

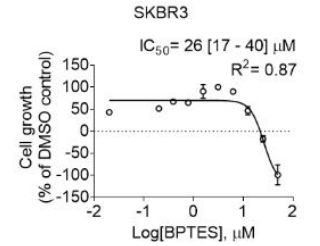

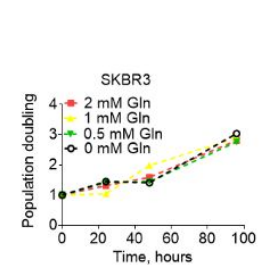

D
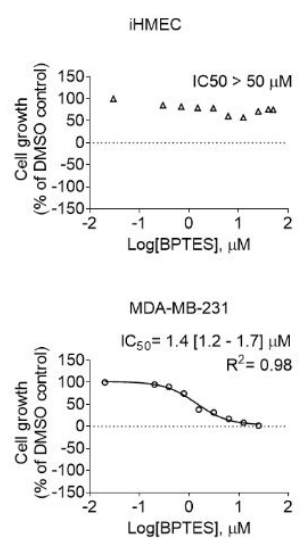

$\mathrm{B}$

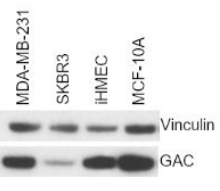

Supplementary Figure S4. Glutamine-deprivation and BPTES effect on cell growth. $A$, Growth response assay of MDA-MB-231, a TNBC cell line, SKBR3, a non-TNBC cell line and the non-tumorigenic MCF-10A cell line to different concentrations of glutamine in the media. $B$, Western blot showing the GLS levels in the cell lines. Growth dose-response assay of BPTES over MDA-MB-231, SKBR3, MCF-10A and hTert-immortalized iHMEC $(C)$ and MDA-MB231 shGFP, shGLS and shGLS expressing GLS2 ectopically $(D)$. On $(C, D)$, the data was normalized and $0 \%$ was set as the number of seeded cells and $100 \%$ as the highest number of cells. Doses bellow the dashed lines indicate the concentrations that lead to cell death (final number of cells after 48 hours is smaller than the number of seeded cells). $\mathrm{IC}_{50}[95 \% \mathrm{CI}]$ and $\mathrm{R}^{2}$ of the adjusted sigmoidal curve are displayed. Graphics in $A, C-D$, each bar represents the mean $\pm \mathrm{SD}$ of $\mathrm{n}=4$ replicates. 

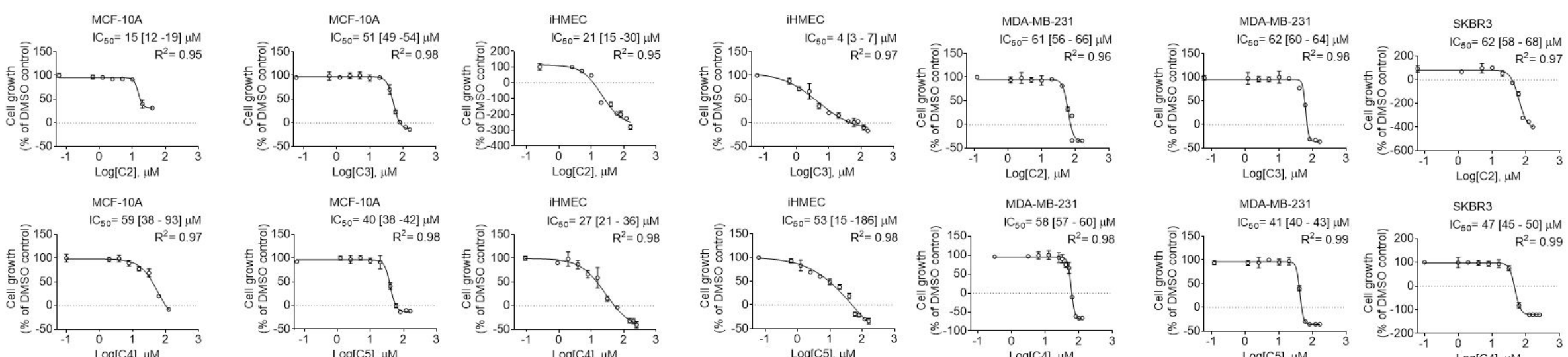

MCF-10A

IHMEC
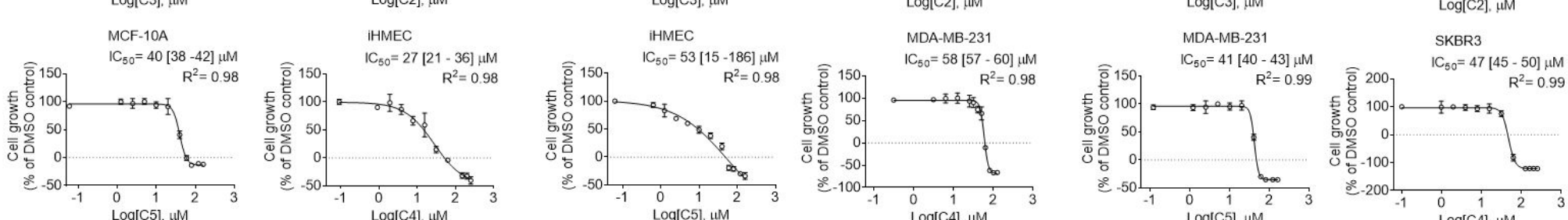

MCF-10A

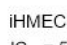

MDA-MB-231
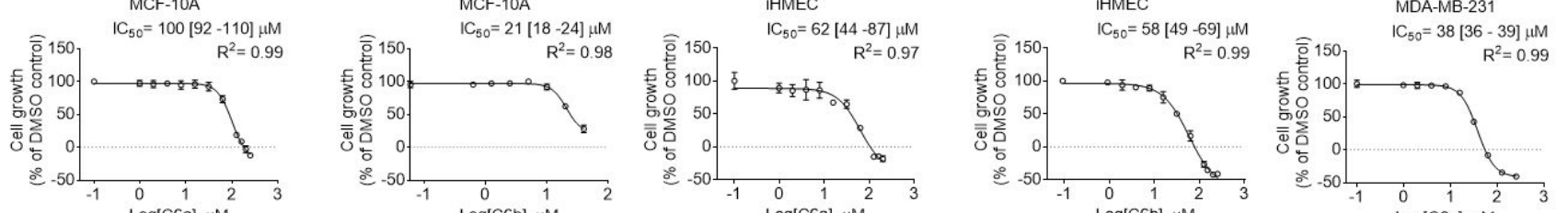

Log[C6b], $\mu \mathrm{M}$
MCF-10A

IHMEC
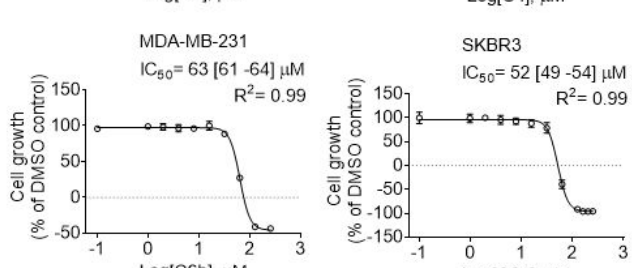

IHMEC

MDA-MB-231
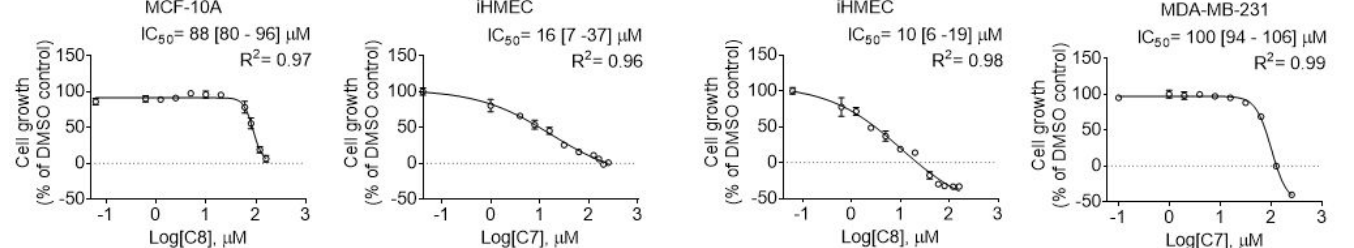

$$
\text { MDA-MB-231 }
$$

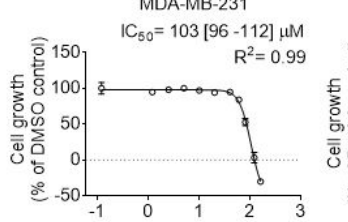

SKBR3

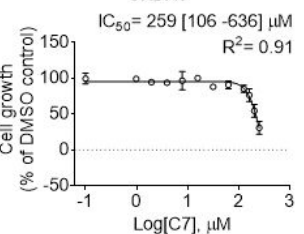

IHMEC
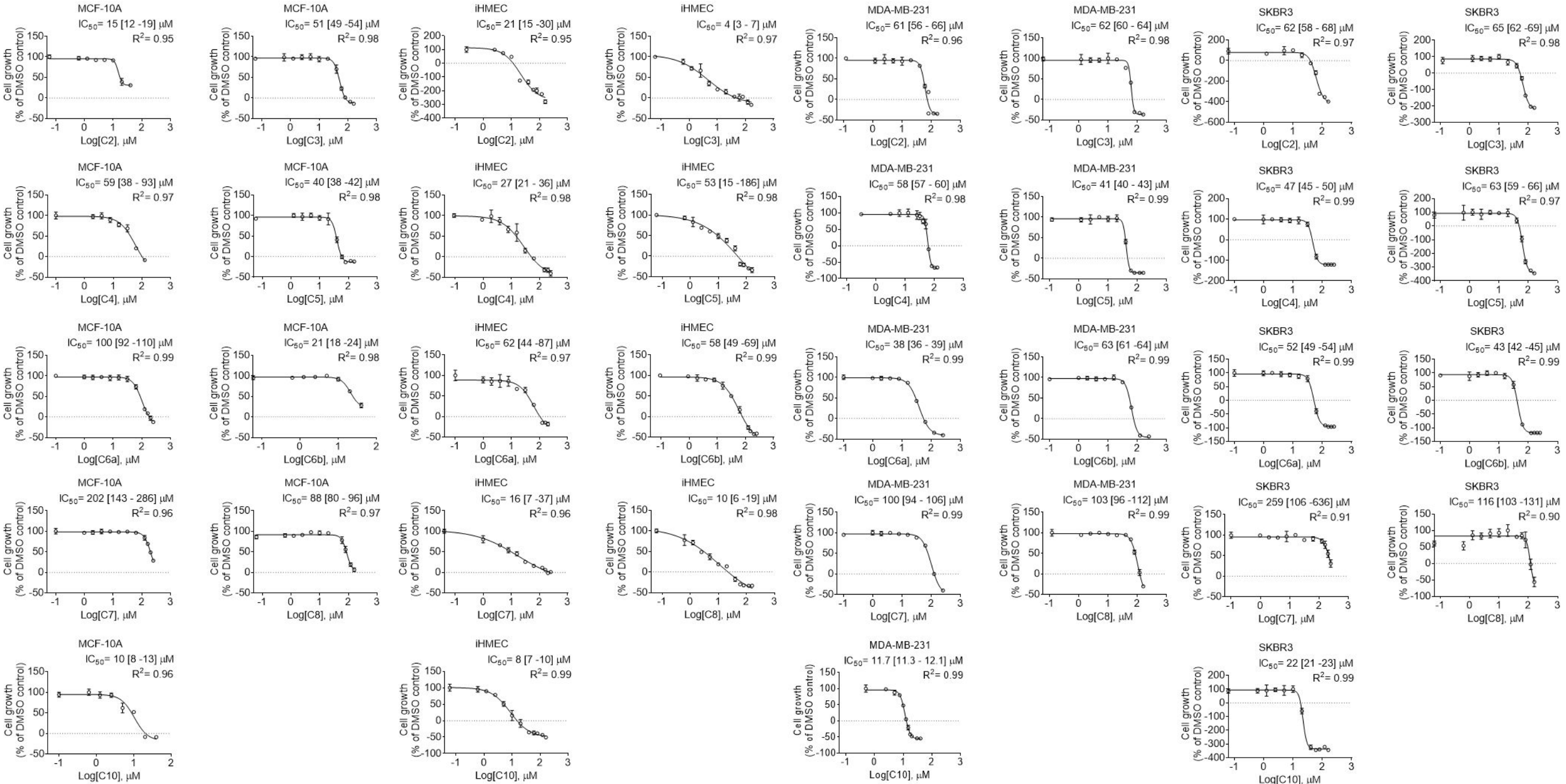

Log[C10], $\mu \mathrm{M}$

Log[C10], $\mu \mathrm{M}$

$\stackrel{0}{0}{ }_{\log }^{1}{ }^{2}{ }^{2}$ 
Supplementary Figure S5. IC 50 curves of C2-C8 and C10 on the MCF-10A, iHMEC, MDA-MB-231 and SKBR3 cell lines growth. Values within the brackets represents an interval confidence of $95 \%$ (CI 95\%). 

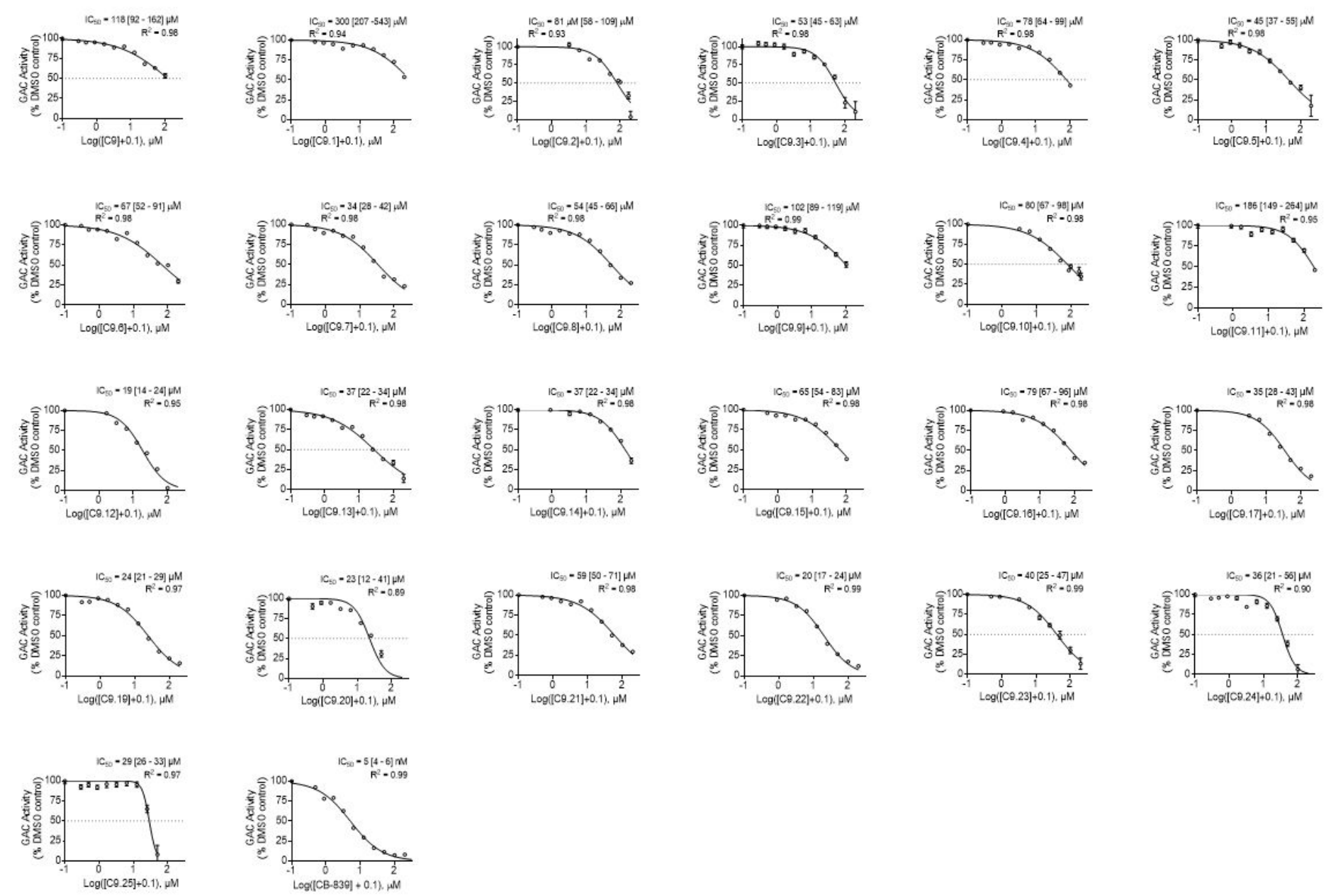

Supplementary Figure S6. $\mathrm{IC}_{50}$ curves of C9 analogues on the GAC enzymatic activity. Values within the brackets represents an interval confidence of $95 \%$ (CI 95\%). 

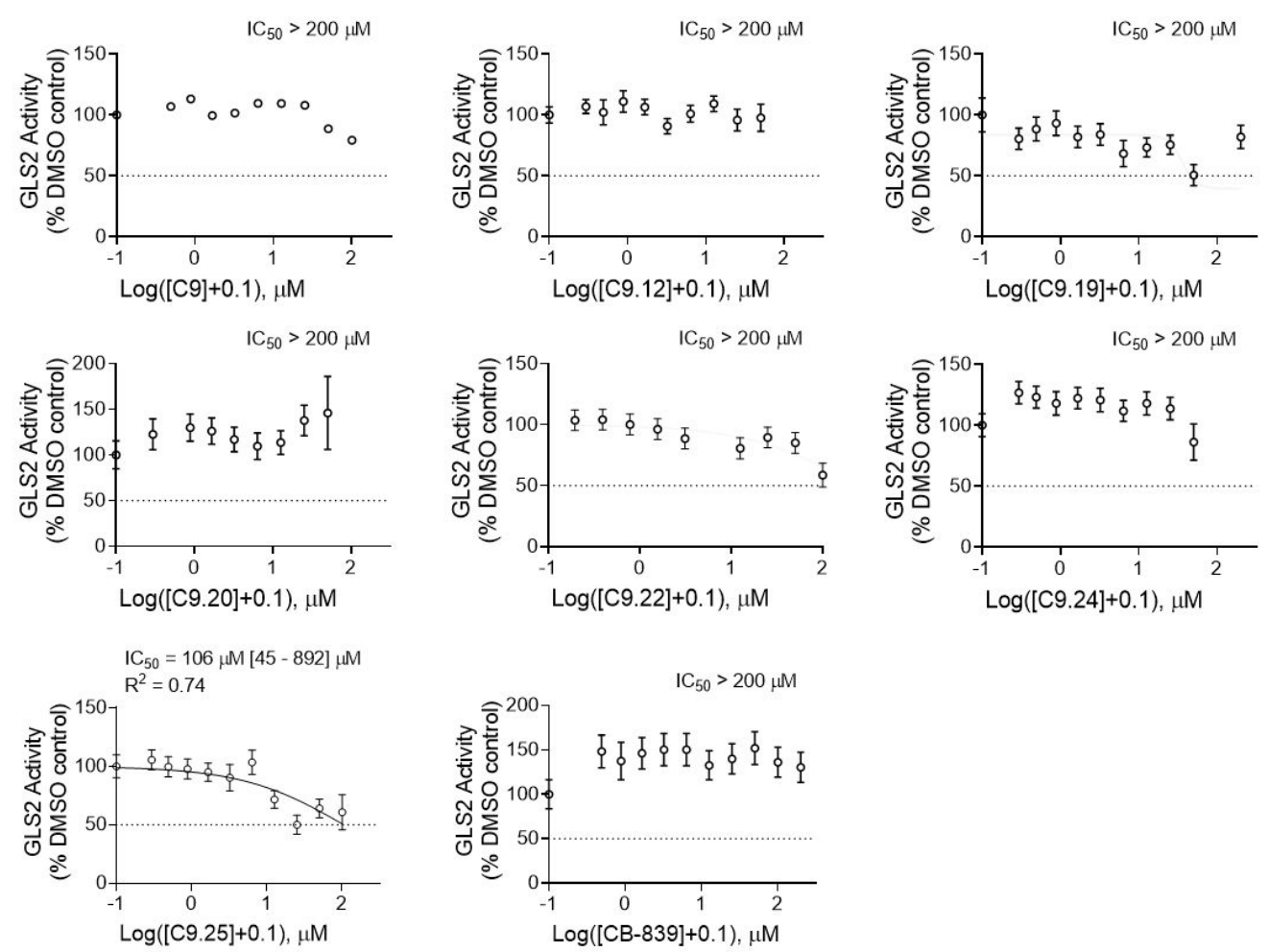

Supplementary Figure S7. $\mathrm{IC}_{50}$ curves of selected C9 analogues on the GLS2 enzymatic activity. Values within the brackets represents an interval confidence of 95\% (CI 95\%). 

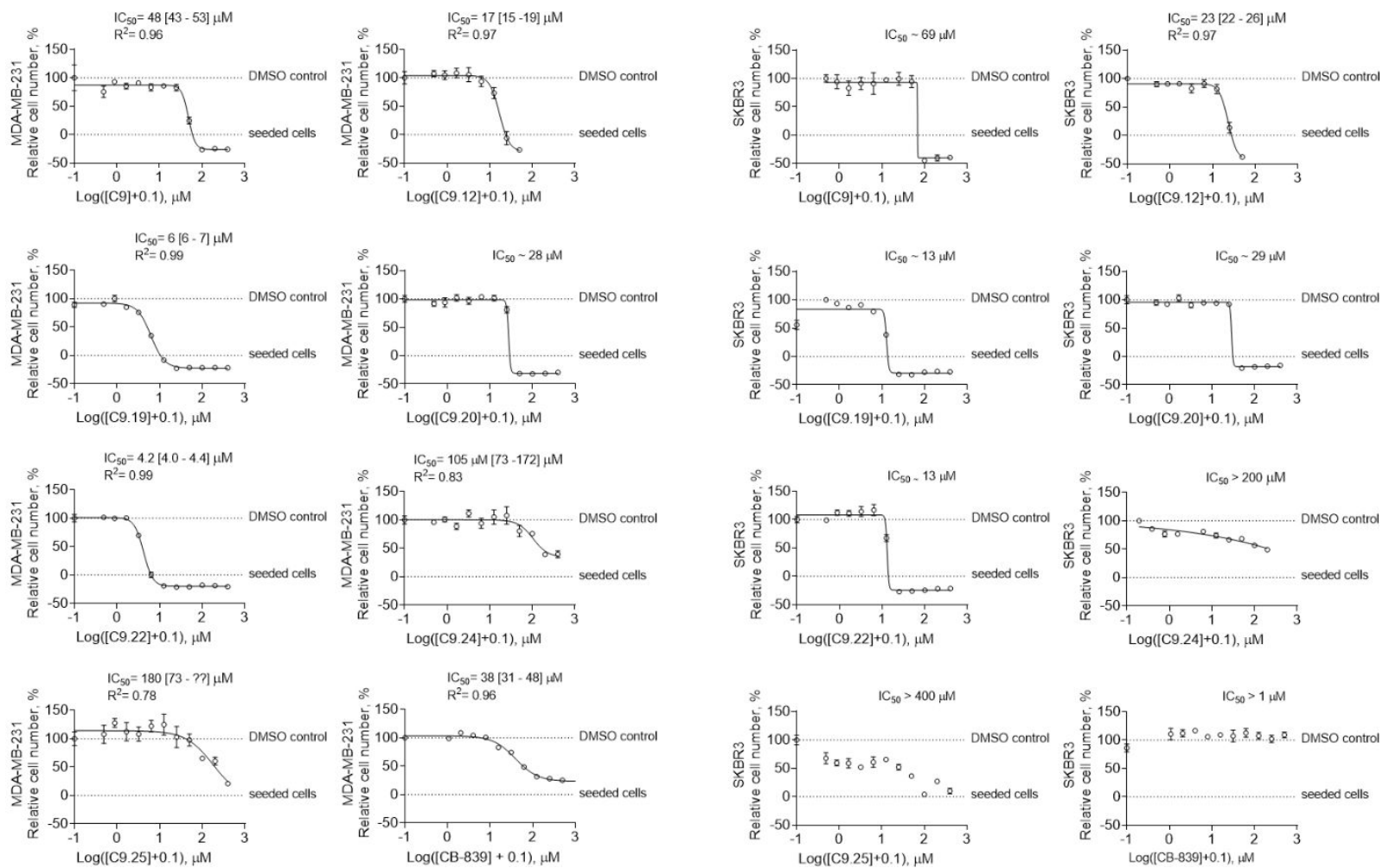

Supplementary Figure S8. IC $_{50}$ curves of selected C9 analogues on the MDA-MB-231 (on the left) and SKBR3 (on the right) cell lines growth. Values within the brackets represents an interval confidence of $95 \%$ (CI 95\%). 


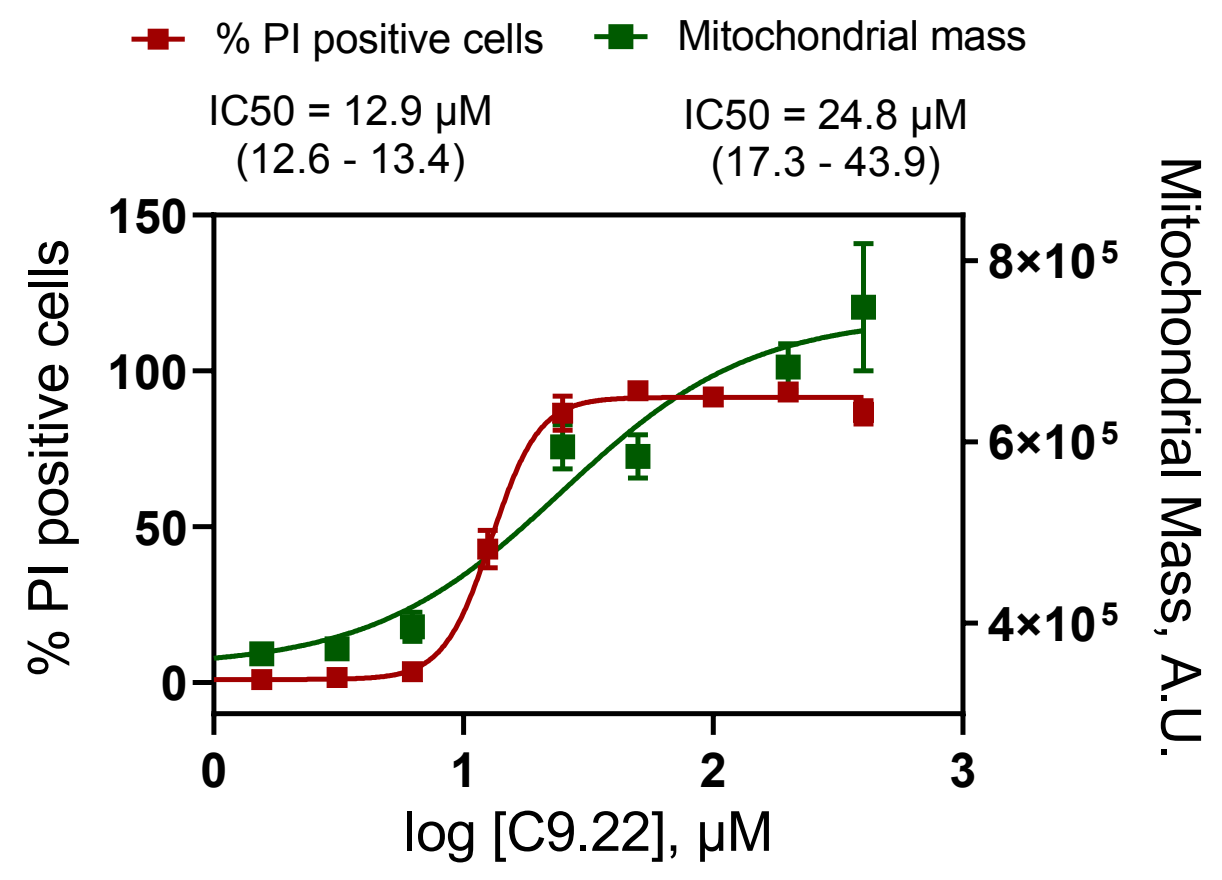

Supplementary Figure 9. $\mathrm{IC}_{50}$ curves of C9.22 on the MDA-MB-231 cell line for necrosis (propidium iodide, PI) and mitochondrial mass stainning (Mitotracker Deep Red; cytotoxicity marker). Values within the brackets represents an interval confidence of 95\%. 
Supplementary Table S1 - Excel file with 6 spreadsheets containing raw and processed HTS data; Provided as a separated file.

Supplementary Table S2 - The final 100 compounds hit list.

\begin{tabular}{|c|c|c|c|c|c|c|}
\hline $\begin{array}{l}\text { Compound } \\
\text { ressuplied }\end{array}$ & $\begin{array}{l}\text { Compoud } \\
\# \text { (100 } \\
\text { final hits) }\end{array}$ & $\begin{array}{l}\text { Chembridge } \\
\text { number }\end{array}$ & $\begin{array}{c}\% \text { inhibition } \\
\text { GAC retest } \\
\text { average }\end{array}$ & $\begin{array}{c}\% \text { inhibition } \\
\text { GDH/Diaphorase }\end{array}$ & $\begin{array}{c}\% \text { inhibition } \\
\text { GAC retest } \\
\text { average - \% } \\
\text { inhibition GDH }\end{array}$ & Cluster \# \\
\hline & 1 & 5251606 & 105.8 & 17.8 & 88.0 & non-clustered \\
\hline & 2 & 5455995 & 88.7 & 0.0 & 88.7 & non-clustered \\
\hline & 3 & 7983133 & 88.6 & 24.7 & 63.9 & non-clustered \\
\hline & 4 & 9109040 & 87.0 & 3.7 & 83.3 & G4 \\
\hline & 5 & 5266583 & 85.8 & 2.3 & 83.5 & non-clustered \\
\hline & 6 & 6985461 & 79.7 & 17.1 & 62.6 & non-clustered \\
\hline$(\mathrm{C} 1)$ & 7 & 7992402 & 73.4 & 0.0 & 73.4 & non-clustered \\
\hline \multirow[t]{3}{*}{$(\mathrm{C} 2)$} & 8 & 9155049 & 72.9 & 2.4 & 70.5 & G11 \\
\hline & 9 & 7975549 & 72.3 & 13.9 & 58.4 & non-clustered \\
\hline & 10 & 5917156 & 72.2 & 11.0 & 61.2 & G17 \\
\hline (C3) & 11 & 7956101 & 69.2 & 0.0 & 69.2 & non-clustered \\
\hline (C4) & 12 & 9125354 & 69.2 & 0.0 & 69.2 & $\mathrm{G} 2$ \\
\hline (C5) & 13 & 7952342 & 68.5 & 0.0 & 68.5 & G7 \\
\hline (C6a) & 14 & 6744277 & 60.2 & 0.0 & 60.2 & G12 \\
\hline$(\mathrm{C} 7)$ & 15 & 5354303 & 60.2 & 0.0 & 60.2 & G5 \\
\hline (C8) & 16 & 7962214 & 58.3 & 0.0 & 58.3 & non-clustered \\
\hline \multirow[t]{12}{*}{ (C6b) } & 17 & 5603967 & 57.3 & 0.0 & 57.3 & G12 \\
\hline & 18 & 7992232 & 57.2 & 0.0 & 57.2 & G15 \\
\hline & 19 & 9038938 & 57.0 & 0.0 & 57.0 & non-clustered \\
\hline & 20 & 9001794 & 56.8 & 0.2 & 56.6 & non-clustered \\
\hline & 21 & 5919129 & 56.7 & 8.2 & 48.4 & G17 \\
\hline & 22 & 5331342 & 54.6 & 11.3 & 43.3 & G6 \\
\hline & 23 & 7832691 & 53.7 & 0.0 & 53.7 & G15 \\
\hline & 24 & 7874229 & 52.8 & 5.0 & 47.7 & non-clustered \\
\hline & 25 & 5141313 & 51.7 & 0.0 & 51.7 & non-clustered \\
\hline & 26 & 6573489 & 51.7 & 12.4 & 39.3 & non-clustered \\
\hline & 27 & 9074873 & 51.7 & 13.3 & 38.4 & G8 \\
\hline & 28 & 7954758 & 51.2 & 0.0 & 51.2 & G3 \\
\hline
\end{tabular}




\begin{tabular}{|c|c|c|c|c|c|c|}
\hline & 29 & 9007772 & 51.0 & 0.0 & 51.0 & non-clustered \\
\hline & 30 & 9082772 & 50.5 & 0.0 & 50.5 & G13 \\
\hline \multirow[t]{2}{*}{ (C9) } & 31 & 9007737 & 50.0 & 0.0 & 50.0 & non-clustered \\
\hline & 32 & 5346214 & 49.3 & 0.0 & 49.3 & G5 \\
\hline \multirow[t]{32}{*}{$(\mathrm{C} 10)$} & 33 & 7951061 & 48.3 & 0.0 & 48.3 & G12 \\
\hline & 34 & 9064772 & 47.3 & 0.0 & 47.3 & G14 \\
\hline & 35 & 7943337 & 46.6 & 0.7 & 45.9 & G16 \\
\hline & 36 & 5211912 & 46.4 & 13.3 & 33.2 & non-clustered \\
\hline & 37 & 9123924 & 46.4 & 0.0 & 46.4 & non-clustered \\
\hline & 38 & 9077494 & 46.4 & 1.2 & 45.1 & G13 \\
\hline & 39 & 6373017 & 46.3 & 0.0 & 46.3 & non-clustered \\
\hline & 40 & 9125395 & 46.1 & 0.0 & 46.1 & $\mathrm{G} 2$ \\
\hline & 41 & 5478253 & 45.9 & 0.0 & 45.9 & non-clustered \\
\hline & 42 & 9139619 & 45.6 & 0.0 & 45.6 & non-clustered \\
\hline & 43 & 5621638 & 45.2 & 0.0 & 45.2 & G12 \\
\hline & 44 & 5319283 & 42.3 & 14.5 & 27.8 & non-clustered \\
\hline & 45 & 7893819 & 41.9 & 0.0 & 41.9 & G13 \\
\hline & 46 & 7797911 & 41.7 & 2.7 & 39.1 & non-clustered \\
\hline & 47 & 7969373 & 41.7 & 12.3 & 29.4 & non-clustered \\
\hline & 48 & 7615470 & 41.6 & 1.4 & 40.1 & non-clustered \\
\hline & 49 & 7946808 & 40.8 & 12.2 & 28.6 & G11 \\
\hline & 50 & 7690736 & 40.7 & 11.4 & 29.2 & non-clustered \\
\hline & 51 & 5348920 & 40.5 & 0.0 & 40.5 & G5 \\
\hline & 52 & 7987960 & 40.3 & 0.0 & 40.3 & non-clustered \\
\hline & 53 & 9064882 & 40.1 & 0.0 & 40.1 & G15 \\
\hline & 54 & 9102535 & 39.4 & 0.0 & 39.4 & G4 \\
\hline & 55 & 7970522 & 38.8 & 0.0 & 38.8 & G7 \\
\hline & 56 & 7953673 & 38.8 & 6.0 & 32.8 & non-clustered \\
\hline & 57 & 7745039 & 38.8 & 5.4 & 33.5 & G8 \\
\hline & 58 & 7950985 & 38.7 & 0.0 & 38.7 & non-clustered \\
\hline & 59 & 9108360 & 38.6 & 2.8 & 35.8 & G13 \\
\hline & 60 & 9014421 & 38.1 & 10.3 & 27.8 & G10 \\
\hline & 61 & 9153561 & 37.8 & 0.2 & 37.6 & non-clustered \\
\hline & 62 & 6946489 & 37.2 & 7.2 & 29.9 & non-clustered \\
\hline & 63 & 5350946 & 37.1 & 0.0 & 37.1 & G5 \\
\hline & 64 & 7257347 & 36.7 & 0.0 & 36.7 & non-clustered \\
\hline
\end{tabular}




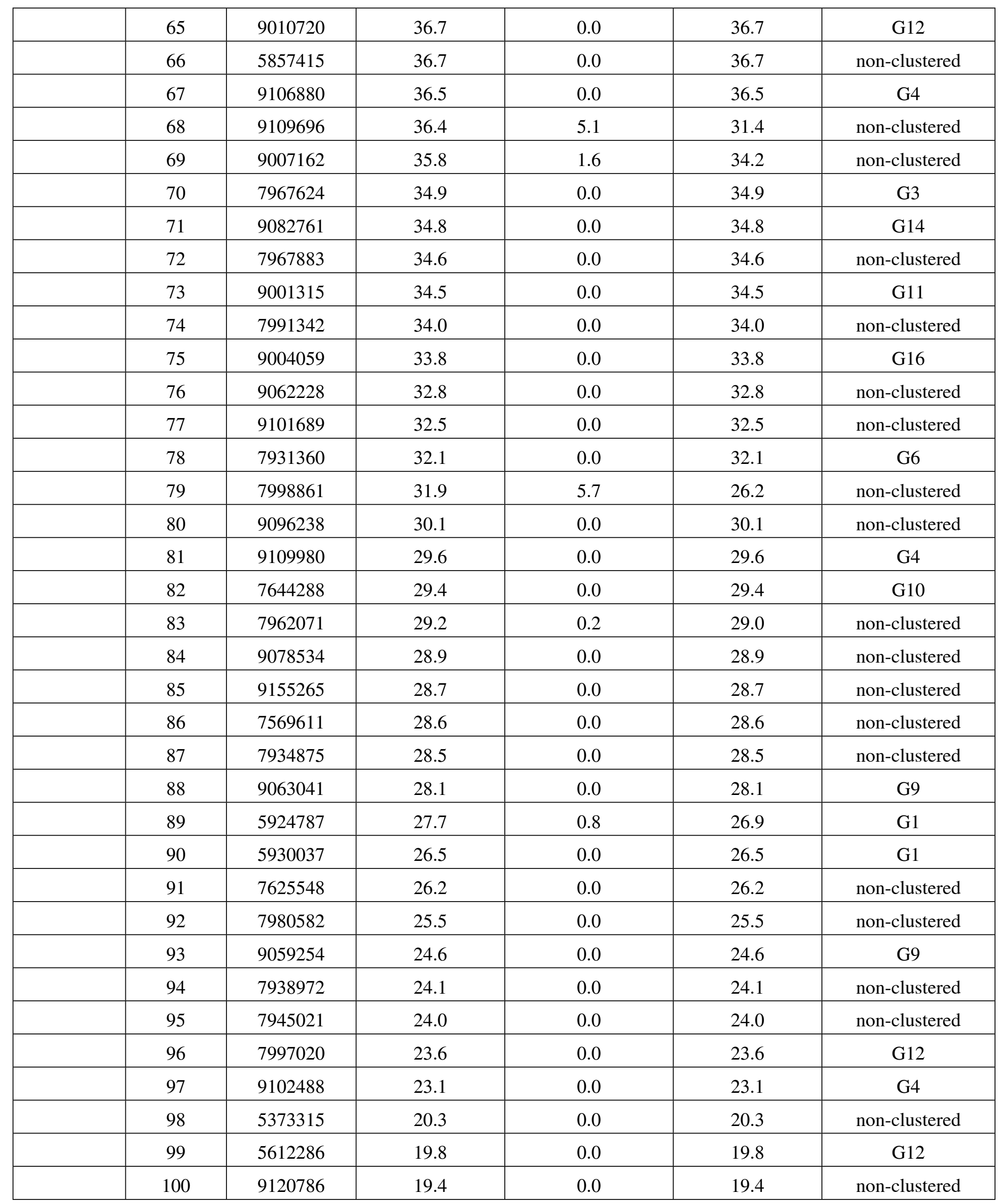


Supplementary Table S3 - Drug-like properties calculated by the OpenBabel. 


\begin{tabular}{|c|c|c|c|c|c|}
\hline & Druglikeness & Mutagenic & Tumorigenic & $\begin{array}{c}\text { Reproductive } \\
\text { Effective }\end{array}$ & Irritant \\
\hline C1 & -4.9666 & none & none & none & none \\
\hline C2 & 2.5939 & none & none & none & none \\
\hline C3 & 2.6252 & none & none & none & none \\
\hline C4 & 4.0452 & none & none & none & none \\
\hline C5 & 0.7881 & none & high & low & high \\
\hline C6a & 2.4671 & none & none & high & low \\
\hline C7 & -1.182 & none & none & none & none \\
\hline C8 & 4.0127 & high & high & high & low \\
\hline C6b & 0.5554 & none & none & high & none \\
\hline C9 & 2.0762 & none & none & none & none \\
\hline C10 & 4.3483 & none & none & none & none \\
\hline 968 & -2.34 & none & none & none & none \\
\hline
\end{tabular}




\begin{tabular}{|c|c|c|c|c|c|}
\hline BPTES & 3.9388 & none & none & none & none \\
\hline CB-839 & -9.1868 & none & none & none & none \\
\hline
\end{tabular}


Supplementary Table S4- In silico safety. ADME and physicochemical profile of the 11 resupplied compounds.

\begin{tabular}{|c|c|c|c|c|c|c|c|c|c|c|c|c|c|c|c|c|c|c|c|c|c|c|}
\hline \multirow[b]{2}{*}{ ID } & \multicolumn{8}{|c|}{ Drug Safety Profiling } & \multicolumn{5}{|c|}{ ADME Profiling } & \multicolumn{9}{|c|}{ PhysChem Profiling } \\
\hline & $\begin{array}{c}\text { P-gp } \\
\text { Substrates }\end{array}$ & $\begin{array}{l}\text { CYP1A2 } \\
\text { Inhibitor }\end{array}$ & $\begin{array}{l}\text { CYP2C9 } \\
\text { Inhibitor }\end{array}$ & \begin{tabular}{c|} 
CYP2C19 \\
Inhibitor
\end{tabular} & $\begin{array}{l}\text { CYP2D6 } \\
\text { Inhibitor }\end{array}$ & $\begin{array}{l}\text { CYP3A4 } \\
\text { Inhibitor }\end{array}$ & Ames & hERG & Caco-2 & PPB & CNS & HIA & $\begin{array}{c}\text { Metabolic } \\
\text { Stability }\end{array}$ & $\log P$ & мw & H-Donors & $\begin{array}{c}\mathrm{H}- \\
\text { Acceptors }\end{array}$ & $\begin{array}{l}\text { Rot. } \\
\text { Bonds }\end{array}$ & Rings & Lipinski & Lead-like & Solubility \\
\hline 968 & Undefined & Undefined & Undefined & Undefined & Undefined & Undefined & Undefined & Undefined & \begin{tabular}{|l} 
Highly \\
permeable
\end{tabular} & $\begin{array}{l}\text { Extensively } \\
\text { bound }\end{array}$ & $\begin{array}{l}\text { Non- } \\
\text { penetrant }\end{array}$ & \begin{tabular}{|l} 
Highly \\
absorbed
\end{tabular} & Undefined & $\begin{array}{l}\text { Very } \\
\text { lipophilic }\end{array}$ & Moderate & Good & Good & Good & Bad & Moderate & Bad & Highly insoluble \\
\hline BPTES & Undefined & Undefined & Undefined & Undefined & Undefined & Undefined & Undefined & Undefined & \begin{tabular}{|l} 
Highly \\
permeable
\end{tabular} & Undefined & \begin{tabular}{|l} 
Non- \\
penetrant
\end{tabular} & \begin{tabular}{|l} 
Highly \\
absorbed
\end{tabular} & Undefined & Optimal & Bad & Good & Good & Bad & Good & Moderate & Moderate & Highly insoluble \\
\hline CB-839 & Undefined & Undefined & Undefined & Undefined & Undefined & Undefined & Undefined & Undefined & \begin{tabular}{|l} 
Highly \\
permeable
\end{tabular} & Undefined & $\begin{array}{l}\text { Non- } \\
\text { penetrant }\end{array}$ & \begin{tabular}{|l} 
Highly \\
absorbed
\end{tabular} & Undefined & Optimal & Bad & Good & Good & Bad & Good & Moderate & Bad & Highly insoluble \\
\hline C1 & Undefined & Undefined & Undefined & Undefined & Undefined & $\begin{array}{l}\text { Non- } \\
\text { inhibitor }\end{array}$ & Undefined & Undefined & \begin{tabular}{|l} 
Highly \\
permeable
\end{tabular} & $\begin{array}{l}\text { Strongly } \\
\text { bound }\end{array}$ & Penetrant & \begin{tabular}{|l} 
Highly \\
absorbed
\end{tabular} & Undefined & Optimal & Good & Good & Good & Good & Good & Good & Good & Insoluble \\
\hline $\mathrm{C} 2$ & Undefined & Undefined & Undefined & Undefined & Undefined & Undefined & Undefined & Undefined & \begin{tabular}{|l} 
Highly \\
permeable
\end{tabular} & Undefined & Penetrant & \begin{tabular}{|l} 
Highly \\
absorbed
\end{tabular} & Undefined & Optimal & Good & Good & Good & Good & Good & Good & Good & Soluble \\
\hline C3 & Undefined & Undefined & Undefined & Undefined & Undefined & Undefined & Undefined & Undefined & \begin{tabular}{|l} 
Highly \\
permeable
\end{tabular} & $\begin{array}{l}\text { Extensively } \\
\text { bound }\end{array}$ & Penetrant & \begin{tabular}{|l} 
Highly \\
absorbed
\end{tabular} & Undefined & Optimal & Good & Good & Good & Good & Good & Good & Good & Highly insoluble \\
\hline $\mathrm{C} 4$ & Undefined & Undefined & Undefined & Undefined & Undefined & Undefined & Undefined & Undefined & \begin{tabular}{|l} 
Highly \\
permeable
\end{tabular} & $\begin{array}{l}\text { Strongly } \\
\text { bound }\end{array}$ & Penetrant & \begin{tabular}{|l} 
Highly \\
absorbed
\end{tabular} & Undefined & Optimal & Good & Good & Good & Good & Good & Good & Good & Soluble \\
\hline C5 & Undefined & Undefined & Undefined & Undefined & Undefined & Undefined & Undefined & Undefined & \begin{tabular}{|l} 
Highly \\
permeable
\end{tabular} & $\begin{array}{l}\text { Moderately } \\
\text { bound }\end{array}$ & Penetrant & \begin{tabular}{|l} 
Highly \\
absorbed
\end{tabular} & Undefined & Optimal & Good & Good & Good & Good & Good & Good & Good & Insoluble \\
\hline C6а & Undefined & Undefined & Undefined & Undefined & $\begin{array}{l}\text { Non- } \\
\text { inhibitor }\end{array}$ & Undefined & Undefined & Undefined & \begin{tabular}{|l} 
Highly \\
permeable
\end{tabular} & $\begin{array}{l}\text { Strongly } \\
\text { bound }\end{array}$ & Penetrant & \begin{tabular}{|l} 
Highly \\
absorbed
\end{tabular} & Undefined & Optimal & Good & Good & Good & Good & Good & Good & Good & Highly insoluble \\
\hline C7 & Undefined & Undefined & Undefined & Undefined & Undefined & Undefined & Undefined & Undefined & \begin{tabular}{|l} 
Moderately \\
permeable
\end{tabular} & $\begin{array}{l}\text { Weakly } \\
\text { bound }\end{array}$ & $\begin{array}{l}\text { Weak } \\
\text { penetrant }\end{array}$ & \begin{tabular}{|l} 
Poorly \\
absorbed
\end{tabular} & Undefined & $\begin{array}{l}\text { Very } \\
\text { hydrophilic }\end{array}$ & Good & Good & Good & Good & Good & Moderate & Moderate & \\
\hline C8 & Undefined & Undefined & Undefined & Undefined & $\begin{array}{l}\text { Non- } \\
\text { inhibitor }\end{array}$ & Undefined & Undefined & Undefined & \begin{tabular}{|l} 
Highly \\
permeable
\end{tabular} & $\begin{array}{l}\text { Extensively } \\
\text { bound }\end{array}$ & Penetrant & \begin{tabular}{|l} 
Highly \\
absorbed
\end{tabular} & Undefined & Optimal & Good & Good & Good & Good & Good & Good & Good & Highly insoluble \\
\hline C6b & Undefined & Undefined & Undefined & Undefined & Undefined & Undefined & Undefined & Undefined & \begin{tabular}{|l} 
Highly \\
permeable
\end{tabular} & $\begin{array}{l}\text { Moderately } \\
\text { bound }\end{array}$ & Penetrant & \begin{tabular}{|l} 
Highly \\
absorbed
\end{tabular} & Undefined & Optimal & Good & Good & Good & Good & Good & Good & Good & Insoluble \\
\hline
\end{tabular}




\begin{tabular}{|c|c|c|c|c|c|c|c|c|l|l|l|l|l|l|l|l|l|l|l|l|l|l|l|l|l|}
\hline C9 & Undefined & Undefined & Undefined & Undefined & Undefined & Undefined & Undefined & Undefined & $\begin{array}{l}\text { Highly } \\
\text { permeable }\end{array}$ & $\begin{array}{l}\text { Moderately } \\
\text { bound }\end{array}$ & Penetrant & $\begin{array}{l}\text { Highly } \\
\text { absorbed }\end{array}$ & Undefined & Optimal & Good & Good & Good & Good & Good & Good & Good & Highly insoluble \\
\hline C10 & Undefined & Undefined & Undefined & Undefined & Undefined & Undefined & Undefined & Undefined & $\begin{array}{l}\text { Highly } \\
\text { permeable }\end{array}$ & $\begin{array}{l}\text { Extensively } \\
\text { bound }\end{array}$ & Penetrant & $\begin{array}{l}\text { Highly } \\
\text { absorbed }\end{array}$ & Undefined & Optimal & Good & Good & Good & Good & Good & Good & Good & Insoluble \\
\hline
\end{tabular}


